\section{Best Clinical Abstract - Tobias Todsen MD PhD}

I am a senior registrar in OtolaryngologyHead \& Neck Surgery and clinical researcher in head and neck ultrasound and medical education. I teach ultrasound at a pre- and postgraduate level, and I am a part of a global health initiative to promote surgeonperformed ultrasound in resource-limited countries. Before my specialization, I received broad clinical training as a Foundation Doctor with 18-month rotation between the Dept. of Internal Medicine, Surgery, and General Medicine at Queen Ingrid's Hospital in Greenland. I have been teaching clinical skills and conducted educational research for more than ten years and have skills within producing educational videos, virtual reality content and online apps with more than 50.000 views yearly: open-surgery.com - Vimeo - Youtube

\section{A randomized multicenter clinical trial comparing transoral ultrasound versus standard of care in the management of patient suspected with peritonsillar abscess}

Tobias Todsen, MD, PhD

Department of Otorhinolaryngology, Head and Neck Surgery \& Audiology, Rigshospitalet, Denmark

\section{Background}

Peritonsillar abscess is a common deep head and neck infection in younger adults primarily treated with needle aspiration and surgical drainage. However, it is difficult to distinguish between a peritonsillar phlegmon and abscess on the clinical ex- amination only, and many patients will have unsuccessful blind needle aspiration attempts performed. This trial aims to explore whether a new technique for transoral ultrasound-guided needle aspiration can decrease the number of unsuccessful needle aspiration and improve the management of patients with peritonsillar infection.

\section{Material \& Methods}

A randomized, controlled multicentre trial were conducted at the departments of Otolaryngology, Head and Neck Surgery at Rigshospitalet, and Odense University Hospital. The patients referred with peritonsillar abscess to the departments were randomized to either standard of care with a clinical examination (control group) or a clinical examination together with pointof-care transoral ultrasound (intervention group). The number of performed needle aspirations and the diagnostic accuracy were evaluated using binary logistic regression and a generalized estimating equation to compare the results between the two groups.

\section{Results}

Ninety-five patients were included in the study (58 at Rigshospitalet and 37 at Odense University Hospital) by 28 different physicians treating the patients. Forty-seven patients were allocated to the intervention group and 48 patients to the control group. The results demonstrated that $31.9 \%$ fewer patients in the intervention group were initially treated with needle aspirations compared to the control group

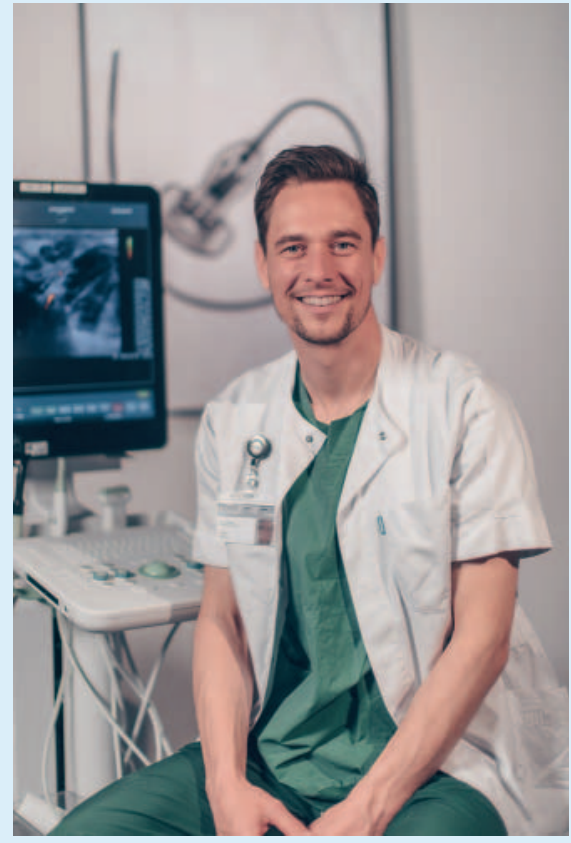

Tobias Todsen MD PhD

$(P<0.01)$, and the total number of needle aspirations decreased from 2.4 to 1.7 mean per patient with the use of transoral ultrasound $(P<0.01)$. The diagnostic accuracy (self-reported) was $80.9 \%$ in the intervention group versus $72.9 \%$ in the control group $(P=0.51)$. Though ultrasound was available in the intervention group, still $40 \%$ of the needle aspiration attempts were performed blind.

\section{Conclusion}

Transoral ultrasound can improve patient management and successfully decrease the number of needle aspiration attempts in patients suspected with peritonsillar abscess. 